\title{
Minimization of counterexamples in SPIN
}

\author{
Paul Gastin, Pierre Moro, and Marc Zeitoun \\ LIAFA, Univ. of Paris 7, Case 7014, \\ 2 place Jussieu, F-75251 Paris Cedex 05, France \\ \{gastin,moro,mz\}@liafa.jussieu.fr
}

\begin{abstract}
We propose an algorithm to find a counterexample to some property in a finite state program. This algorithm is derived from SPIN's one, but it finds a counterexample faster than SPIN does. In particular it still works in linear time. Compared with SPIN's algorithm, it requires only one additional bit per state stored. We further propose another algorithm to compute a counterexample of minimal size. Again, this algorithm does not use more memory than SPIN does to approximate a minimal counterexample. The cost to find a counterexample of minimal size is that one has to revisit more states than SPIN. We provide an implementation and discuss experimental results.
\end{abstract}

\section{Introduction}

Model-checking is used to prove the correctness of properties of hardware and software systems. When the model is incorrect, locating errors is important to provide hints on how to correct either the system or the property to be checked. Model checkers usually exhibit counterexamples, that is, faulty execution traces of the system. The simpler the counterexample is, the easier it will be to locate, understand and fix the error. A counterexample can mean that the abstraction of the system (formalized as the model) is too coarse; several techniques can be used to refine the model, guided by the counterexample found by the modelchecker $[3,1,7]$. The refinement stage is done manually or automatically. In any case, it is important to compute small counterexamples (ideally of minimal size) in case the property is not satisfied: they are easier to understand, they can be processed more rapidly by automatic tools, and thus they make it possible to correct underlying errors more easily.

It is well-known that verifying whether a finite state system $\mathcal{M}$ satisfies an LTL property $\varphi$ is equivalent to testing whether a Büchi automaton $\mathcal{A}=$ $\mathcal{A}_{\mathcal{M}} \cap \mathcal{A}_{\neg \varphi}$ has no accepting run [11], where $\mathcal{A}_{\mathcal{M}}$ is a Kripke structure describing the system and $\mathcal{A}_{\neg \varphi}$ is a Büchi automaton describing executions that violate $\varphi$. It is easy, in theory, to determine whether a Büchi automaton has at least one accepting run. Since there is only a finite number of accepting states, this problem is equivalent to finding a reachable accepting state and a loop around it. A counterexample to $\varphi$ in $\mathcal{M}$ can then be given as a path $\rho=\rho_{1} \rho_{2}$ in the Büchi automaton, where $\rho_{1}$ is a simple (loop-free) path from the initial state to an accepting state, and $\rho_{2}$ is a simple loop around this accepting state (see 


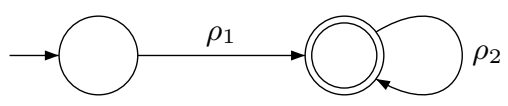

Fig. 1. An accepting path in a Büchi automaton

Figure 1). The model-checker SPIN $[9,8]$ can find counterexamples by exploring on the fly the synchronized product of the system and the property. Our goal is to find short counterexamples while sparing memory. The first trivial remark is that we can reduce the length of a counterexample if we do not insist on the fact that the loop starts from an accepting state. Hence, we consider counterexamples of the form $\rho=\rho_{1} \rho_{2} \rho_{3}$ where $\rho_{1} \rho_{2}$ is a path from the initial state to an accepting state, and $\rho_{3} \rho_{2}$ is a simple loop around this accepting state (see Figure 2). A minimal counterexample can then be defined as a path of this form, such that the length of $\rho$ is minimal.

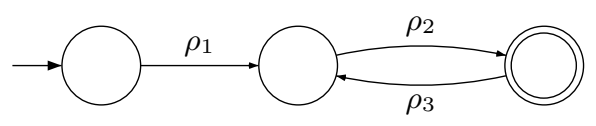

Fig. 2. An accepting path in a Büchi automaton

Finding a counterexample, even of minimal size, can of course be done in polynomial time using minimal paths algorithms based on breadth-first traversals. However, breadth-first traversals are not well-suited to detect loops. Moreover, the model of the system frequently comes from several components working concurrently, and the resulting Büchi automaton can be huge. Therefore, memory is a critical resource and, for instance, we cannot afford to store the minimal distance between all pairs of states. Therefore, we retain SPIN's approach and we use a depth-first search-like algorithm [10,5]. Depth-first traversals are well suited to detect loops, but they are not adapted for computing distances between states, which makes the problem more difficult than it first appears.

With this approach, there are actually two difficulties: the first one is to find one counterexample, the second one is to find a small counterexample, and ideally a minimal one.

SPIN has an option to reduce the size of counterexamples it finds. Yet, it does not provide the smallest one and results frequently remain too large and difficult to read, even when considering simple systems. For instance, on a natural liveness property on Dekker's mutual exclusion algorithm, SPIN provides a counterexample with 173 transitions. In this case, it is not difficult to see that an error occurs after 23 steps. The reason is that SPIN's algorithm for reducing the size of counterexamples misses lots of them and therefore fails to find the shortest one. Our contribution is the following:

- We propose an algorithm to find a counterexample of a Promela model in linear time. This algorithm is derived from SPIN's, but finds a counterex- 
ample faster than SPIN does. Moreover, compared with SPIN's algorithm, it only requires one additional bit per state stored.

- We propose another algorithm to compute a counterexample of minimal size, once a first counterexample has been found. This algorithm does not use more memory than SPIN does with option -i when trying to reduce the size of counterexamples. The cost of finding the shortest counterexample is to revisit more states than SPIN does. However, the algorithm can actually output a sequence of counterexamples of decreasing length found during its execution and can be stopped at any time. The algorithm is also well suited for bounded-model checking: given a maximal size of the counterexamples to be found, it returns one of the smallest such counterexamples, if any.

- We have implemented a version of the last algorithm whose results are indeed much smaller than those given by SPIN. For instance, for Dekker's algorithm, it actually finds the 23 states counterexample.

- We finally propose other improvements to SPIN's algorithm.

The paper is organized as follows. In Section 2, we describe the algorithm to find a first counterexample and we prove its correctness. However, there is no guarantee that this counterexample is of minimal size. In Section 3, we present an algorithm finding a minimal counterexample. While explaining these algorithms, we exhibit various problems that may arise when computing a counterexample with the current SPIN algorithm. An implementation and experimental results are described in Section 4.

\section{Finding the first counterexample}

Let $\mathcal{A}=\left(S, E, s_{1}, F\right)$ be a Büchi automaton where $S$ is a finite set of states, $E \subseteq S \times S$ is the transition relation, $s_{1} \in S$ is the initial state and $F \subseteq S$ is the set of accepting states. Usually transitions are labeled with actions but since these labels are irrelevant for the emptiness problem, they are ignored in this paper. In pictures, the initial state is marked with an ingoing edge and accepting states are doubly circled. If a state has $k$ outgoing transitions, we number them from 1 to $k$. Transitions from a state will be considered by the algorithms in the order given by their labels.

A path in an automaton is a sequence of states $\gamma=t_{1} t_{2} \cdots t_{k}$ (also denoted $t_{1}, t_{2}, \ldots, t_{k}$ ) such that for all $i<k$ there is a transition from $t_{i}$ to $t_{i+1}$. We call $k$ the length of $\gamma$, and we denote it by $|\gamma|$. The empty path, with no state, is denoted by $\varepsilon$ and it has length 0 . We say that $\gamma$ is simple if $t_{i} \neq t_{j}$ for all $i \neq j$.

A loop is a path $t_{1} t_{2} \cdots t_{k}$ with $t_{k}=t_{1}$. A loop is accepting if it contains an accepting state. A loop $t_{1} t_{2} \cdots t_{k}$ is a cycle if $t_{1} t_{2} \cdots t_{k-1}$ is a simple path.

An accepting path, or counterexample, is of the form $\gamma=s_{1} \cdots s_{k} \cdots s_{k+\ell}$ where $s_{1} \cdots s_{k}$ is a path starting from the initial state and $s_{k} \cdots s_{k+\ell}$ is an accepting loop. Abusing the language, we say that $\gamma$ is a simple accepting path if in addition $s_{1} \cdots s_{k} \cdots s_{k+\ell-1}$ is simple.

In this section, we describe an algorithm finding the first counterexample. It is similar to the nested DFS described in $[9,5,2,10]$, with an improvement that 
avoids revisiting some states unnecessarily. This improvement is also useful when minimizing the size of the counterexample.

Algorithm 1 uses 4 colors to mark states: white $<$ blue $<$ red $<$ black. (We also mark states in grey, but this is just for simplifying the proof.) The color of a state can only increase. At the beginning, all states are white and the algorithm DFS_blue is called on the initial state $s_{1}$.

Two DFSs alternate, the blue and red ones. The blue DFS is used to locate reachable accepting states and to start red DFSs from these accepting states in postfix order with respect to the covering tree defined by the blue DFS. A red DFS starts (and interrupts the blue one) whenever one pops an accepting state in the blue DFS. A red DFS only visits blue states, that is states already visited by the blue DFS. We will show that if a red DFS initiated from an accepting state $r$ terminates without finding a counterexample then no state reachable from $r$ may be part of an accepting path. Hence, the color of all states reachable from $r$ may be set to black. This is the purpose of the black DFS.

The DFSs used define, at any time, a current path from the initial state to the current state. For convenience, this current path is stored in a global variable $\mathrm{cp}$. Actually, this is not necessary with our recursive presentation, since it may be obtained as a by-product of the execution stack when the counterexample is found. (For efficiency, SPIN uses an iterative implementation of the DFS, and stores the current path in a global variable.)

Each state $s \in S$ is represented by a structure and the algorithm requires the following additional fields. The extra cost of these data is only 3 bits for each state, while the nested DFS implemented in SPIN only needs 2 bits per state.

- Color color initially white.

- Boolean is_in_cp initially false. This flag is used to test in constant time whether a state belongs to the current path.

When we write for all $t \in E(s)$ in the algorithms (see e.g. Algorithm 1), we assume that the successors $\{t \in S \mid(s, t) \in E\}$ of $s$ are returned in a fixed order, which is in particular the same in DFS_blue and DFS_red. This fact is important for the correctness of Algorithm 1. We establish simultaneously the following invariants.

Lemma 1. (1) Invariant for DFS_blue: no black state is part of a simple accepting path and all states reachable from a black state are also black.

(2) Invariant for DFS_red initiated from DFS_red( $r)$ with $r \in F$ : either no state reachable from $r$ is part of a simple accepting path, or there is a simple accepting path going through $r$ and using no black or grey state.

Proof. (1) During DFS_blue(s), if we execute line 8 then all successors of $s$ are black and the result is clear by induction. Now, assume that we execute line 11. Then DFS_red $(s)$ was executed completely and the color of $s$ is grey. Using (2) (with $r=s$ ) we deduce that no state reachable from $s$ is part of a simple accepting path. Hence, after executing DFS_black $(s)$, the invariant is still satisfied. 


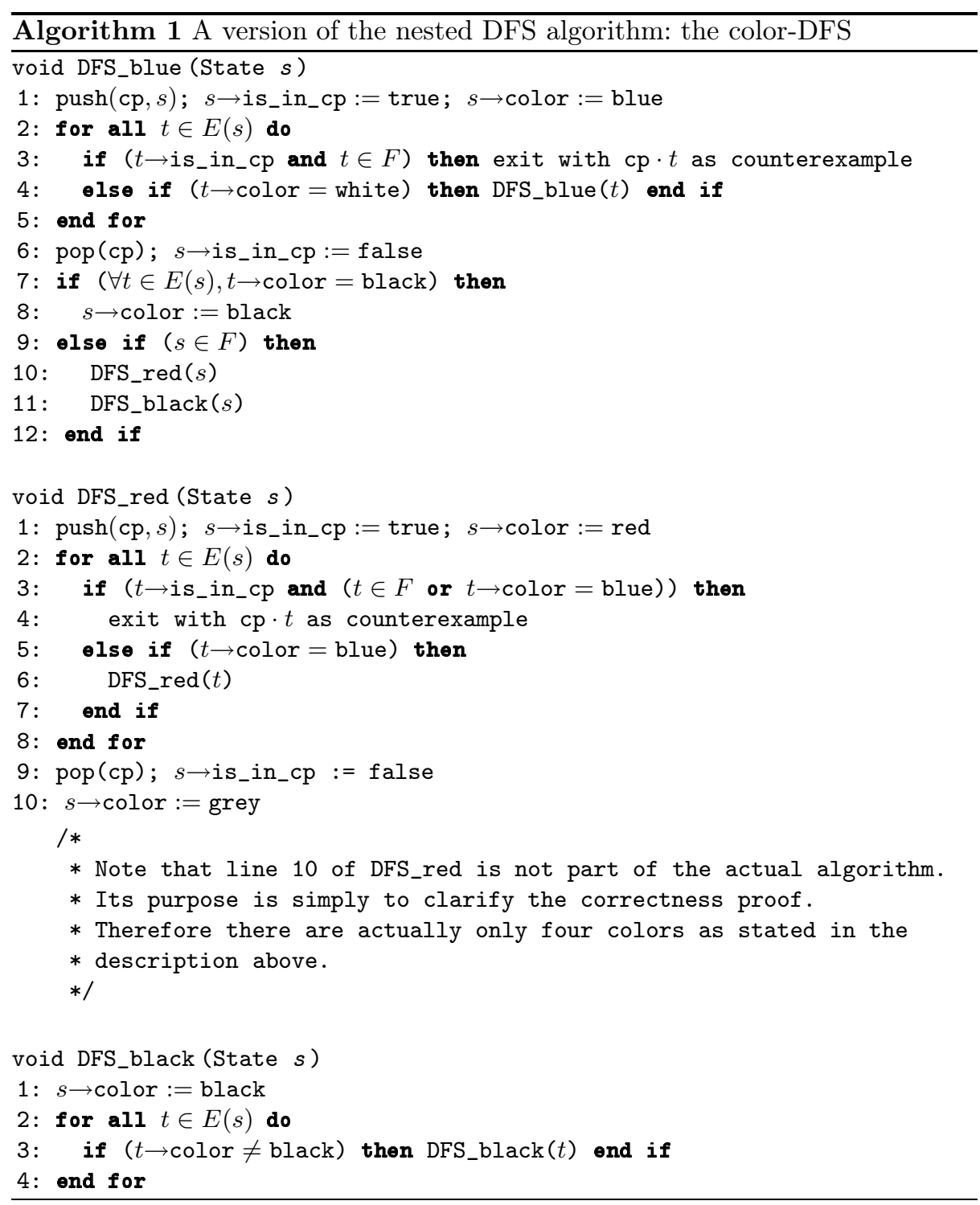

(2) This is the difficult part. First, note that when entering DFS_red $(r)$ there are no grey states and we get property (2) directly from (1). Now, this invariant may only be affected by the execution of line 10 inside some DFS_red $(s)$. When executing this statement, all successors of $s$ are either black, grey, or red. Note that a red successor of $s$ is necessarily on the current path between $r$ and $s$ since the states on $\mathrm{cp}(r)$ are still blue, where $\mathrm{cp}(r)$ is the current path when DFS_red $(r)$ was called. 
Assume that there exists a simple accepting path $\alpha$ going through $r$ and using no black or grey state. Note that all paths using no black state and going from $r$ to an accepting state must $\operatorname{cross} \mathrm{cp}(r) \cdot r$. This is due to the postfix order of the calls DFS_red $(t)$ for $t \in F$. Since we can reach an accepting state, following $\alpha$ from $r$, unwinding $\alpha$ once if necessary, we get a path $\beta$ from $r$ to $c p(r) \cdot r$ using no black or grey state. The path $\mathrm{cp}(r) \cdot \beta$ is a simple accepting path using no black or grey state.

If $s \notin \beta$ then the invariant still holds after setting the color of $s$ to grey in line 10. Assume now that $s \in \beta$ and let $t$ be the successor of $s$ on the path $\beta$. The color of $t$ must be red. Let $v$ be the last state of $\beta$ whose color is red and write $\beta=\beta_{1} v \beta_{2}$. Since the color of $v$ is red, it is on the current path between $r$ and $s$ and $\mathrm{cp}(r) \cdot r$ is a prefix of $\mathrm{cp}(v) \cdot v$. Therefore, $\mathrm{cp}(\mathrm{v}) \cdot v \beta_{2}$ is a simple accepting path using no grey or black states and does not contain $s$. Hence, the invariant still holds after setting the color of $s$ to grey at line 10 .

Remark 1. One can prove that if a call DFS_red $(r)$ with $r \in F$ terminates without finding a counterexample, then all states reachable from $r$ are black or grey. Therefore, at line 10 of DFS_red $(s)$, we could set the color of $s$ to black directly and remove line 11 (the call to DFS_black) in DFS_blue. This modification is fine if we are only interested in finding the first counterexample. But when the color of some state $s$ is set to grey, then we do not know whether $s$ is part of a counterexample or not. In other words, one can deduce that a grey state cannot be part of a counterexample only when the initial call DFS_red $(r)$, with $r \in F$, terminates. In order to avoid revisiting unnecessarily some states, the minimization algorithm presented in Section 3 can use the fact that a black state cannot be part of a counterexample. This is why we do not use this modification.

Since the algorithm visits a state at most 3 times, Algorithm 1 terminates. Moreover, one gets as a corollary of Lemma 1 the following statement.

Proposition 1. If a Büchi automaton $\mathcal{A}$ admits a counterexample, then Algorithm 1 finds a counterexample on input $\mathcal{A}$.

\subsection{Comparison with SPIN's algorithm}

The difference between our algorithm and SPIN's is that SPIN does not paint states in black to avoid unnecessary revisits of states. More precisely, in SPIN's algorithm, lines 7 to 12 of DFS_blue are replaced with

$$
\text { if } s \in F \text { then } r:=s \text {; DFS_red(s) endif }
$$

where $r$ is a global variable used to memorize the origin of the red DFS. To illustrate the benefit of black states, consider the automaton below. Recall that the transition labels indicate in which order successors are considered by the DFSs. With SPIN's algorithm, the large tree is visited twice. The first visit is started with DFS_blue(2) and the second one with DFS_red(3). With our algorithm, when DFS_blue(2) terminates, state 2 is black. Indeed, DFS_blue is 
called recursively on each state of the tree accessible from state 2 . All leaves of this tree, which have no successor, are marked black at lines 7-8, and this propagates back to state 2 . Therefore the tree will not be revisited by DFS_red(3).

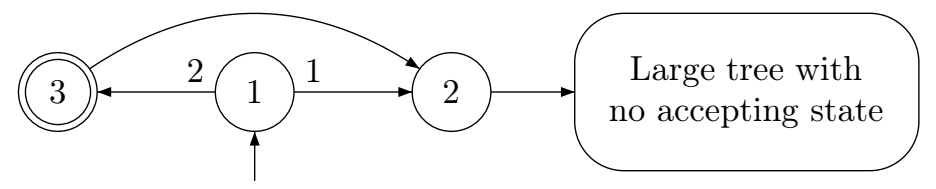

\section{Finding a minimal counterexample}

To find a minimal counterexample, we use a depth-first search [4] which does not necessarily stop when it reaches a state already visited. Indeed, reaching a state $s$ with a distance to the initial state $s_{1}$ smaller than for the previous visit of $s$ may lead to a shorter counterexample.

Therefore, in addition to the fields used in Algorithm 1, each state has an integer field depth, storing the smallest length of current paths on which that state occurred. This field remains infinite as long as the state has not been visited, and it can only decrease during the algorithm. We also use an additional variable mce, a stack of states containing the minimal counterexample found so far. It is initially empty. At the end of the algorithm, it will contain a minimal counterexample of the whole automaton.

\subsection{SPIN's algorithm}

The current algorithm implemented in SPIN to find a small counterexample is a variation of the nested DFS algorithm [10]. It carries on the visit below a state either if the state is new or if it is found more quickly than during the previous visits. (And, before popping an accepting state, it looks for a loop from that state.) This algorithm cannot guarantee to find a minimal counterexample. The reason is that, after finding the first counterexample, SPIN backtracks whenever it reaches a state with a path longer than the stored distance to the initial state. This is due to the false intuition that using a longer path will never yield a shorter counterexample. There are two cases where this is not appropriate and the minimal counterexample is missed. The following examples illustrate these two cases. As before, transition labels indicate in which order they are visited.

In the automaton of Fig. 3, the first counterexample found is $s_{1} s_{2} s_{3} s_{4} s_{5} s_{6} s_{3}$.

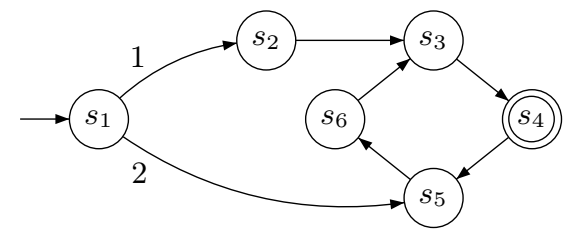

Fig. 3. Missing the minimal counterexample: case 1 
After this visit, the state depths are set as follows: $\left(s_{1}, 1\right),\left(s_{2}, 2\right),\left(s_{3}, 3\right)$, $\left(s_{4}, 4\right),\left(s_{5}, 5\right),\left(s_{6}, 6\right)$. SPIN's algorithm then backtracks and $s_{5}$ is reached from $s_{1}$ with depth 2. Since this is smaller than the previous depth of $s_{5}$ the visit proceeds to $s_{6}$ which is reached now at depth 3 , and then to $s_{3}$, reached at depth 4 . But 4 is greater than the previous depth of $s_{3}$ and SPIN's algorithm would backtrack missing the shortest counterexample which is $s_{1} s_{5} s_{6} s_{3} s_{4} s_{5}$.

The second case is when an accepting state is on the current path. Then, even if no depth was reduced after finding the first counterexample, one should revisit already visited states. An example is shown in Fig. 4.

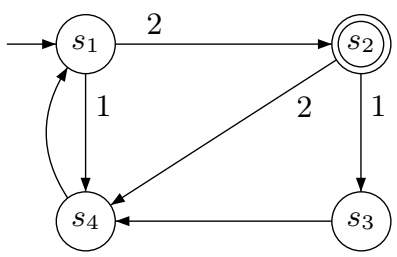

Fig. 4. Missing the minimal counterexample: case 2

The first counterexample found (during the second depth-first search from $s_{2}$ ) is $s_{1} s_{2} s_{3} s_{4} s_{1}$ and the state depths are $\left(s_{1}, 1\right),\left(s_{2}, 2\right),\left(s_{3}, 3\right),\left(s_{4}, 2\right)$. Now, when we reach $s_{4}$ from $s_{2}$ with the current path $s_{1} s_{2} s_{4}$, no depth has been reduced and again SPIN's algorithm would backtrack missing the shortest counterexample which is $s_{1} s_{2} s_{4} s_{1}$. In this case, the relevant length that was reduced is the length from the accepting state $s_{2}$ to $s_{4}$ (from 2 to 1 ). Because memory is the most critical resource, it is not possible to store the length from all accepting states to each state. Therefore, we have to revisit states already visited.

To cope with these cases, Algorithm 2 has two operating modes: a normal one where several criteria can make the algorithm backtrack, and a more careful one, where the visit can only stop when either the current path loops, or becomes longer than the size of the minimal counterexample found so far. In this mode, states may be revisited several times. If the algorithm enters in careful mode while pushing a state $s$ on the current path, it remains in this mode until that occurrence of $s$ is popped off the current path.

In the example of Fig. 3, we would switch to careful mode at lines 11-12 of Algorithm 2 when visiting $s_{5}$ for the second time, because the field $s_{5} \rightarrow$ depth gets reduced. In the example of Fig. 4, we would switch to careful mode at lines $7-8$ when visiting $s_{2}$, an accepting state.

The important fact is that being careful only in these two situations is sufficient to catch a minimal counterexample.

\subsection{An algorithm finding the minimal counterexample}

Algorithm 2 is again presented by a recursive procedure which tags states while visiting them. Its first argument is the state to be visited. Its second argument 
is the mode, initially normal, used for the visit. When we detect that some counterexample might be missed in that mode, we switch to the careful mode by calling the procedure with careful as the second argument. The mode could be implemented as a global variable, which saves memory. Making it an argument of the procedure yields a simpler presentation of the algorithm.

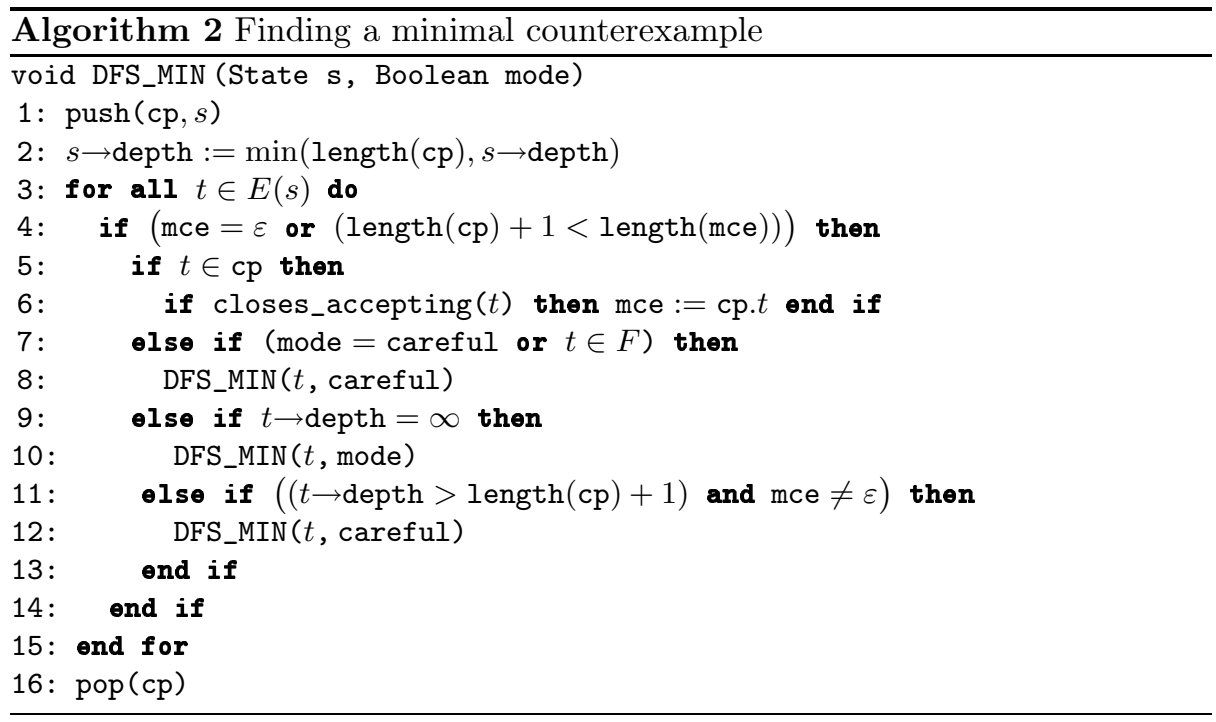

In the description of Algorithm 2, we use the following functions:

- int length(p) returns the length of the path $\mathrm{p}$ (i.e., its number of states). Since we only use it with $\mathrm{cp}$ and mce as arguments, one can maintain their lengths in two global variables, hence we may assume that this call requires $O(1)$-time.

- Boolean closes_accepting $(t)$ returns true iff $\mathrm{cp} \cdot t$ is an accepting path (assuming that $\mathrm{cp}$ itself is not accepting). To implement this function, one can use another stack of states recording, for each state $s$ of the current path $\mathrm{cp}$ the depth in $\mathrm{cp}$ of the last accepting state of $\mathrm{cp}$ located before $s$. For instance, if the current path is $\left[s_{1}, s_{2}, s_{3}, s_{4}, s_{5}, s_{6}\right]$ and only $s_{2}, s_{5}$ are accepting, then this stack contains $[0,2,2,2,5,5]$ (where 0 means that there is no accepting state). The function closes_accepting can then be implemented:

- in $O(1)$-time if we accept to store the depth of each state on the current path. To check that a state closing a cycle creates an accepting cycle, one checks that the depth of its occurrence on the current path is smaller than the depth of the last accepting state on the current path.

- in $O(n)$-time otherwise, where $n$ is the length of the current path. Nevertheless, the additional stack still gives useful information to avoid visiting 
the current path. For instance, if $s \rightarrow$ depth (which will be smaller than the depth of $s$ in $\mathrm{cp}$ ) is larger than the depth of the last accepting state on the current path, or if there is no accepting state on it, we know that $s$ does not close an accepting path.

\subsection{Correctness of the algorithm}

To prove that Algorithm 2 is correct, we introduce the lexicographic ordering on paths starting from the initial state of the automaton. Recall that if a state has $k$ outgoing transitions, they are labeled from 1 to $k$ according to the order in which they will be processed by the algorithm. Let $\lambda: S \times S \rightarrow \mathbb{N}$ assigning to each edge its labeling. We extend $\lambda$ to paths starting at $s_{1}$ by letting $\lambda\left(s_{1}\right)=$ $\varepsilon$ and $\lambda\left(s_{1}, s_{2}, \ldots, s_{n}\right)=\lambda\left(s_{1}, s_{2}\right) \lambda\left(s_{2}, s_{3}\right) \cdots \lambda\left(s_{n-1}, s_{n}\right)$. If $\gamma$ and $\gamma^{\prime}$ are two paths starting at $s_{1}$, we say that $\gamma$ is lexicographically smaller than $\gamma^{\prime}$, denoted $\gamma \prec_{\text {lex }} \gamma^{\prime}$, if $\lambda(\gamma)$ is lexicographically smaller than $\lambda\left(\gamma^{\prime}\right)$ (with the usual order over $\mathbb{N}$ ). We let $\gamma \preceq$ lex $\gamma^{\prime}$ iff $\gamma \prec_{\text {lex }} \gamma^{\prime}$ or $\gamma=\gamma^{\prime}$.

The first observation is that the algorithm discovers paths in increasing lexicographic order. In other words, each call to DFS_MIN makes the current path greater in the lexicographic ordering.

Lemma 2. Let $\alpha$ and $\beta$ be the values of $\mathrm{cp}$ after two consecutive executions of line 1 of Algorithm 2. Then, $\alpha \prec_{\text {lex }} \beta$.

Proof. First observe that the test at line 5 guarantees that the current path cp remains simple: DFS_MIN will not be called on a state that would close the current path. Let $\alpha=s_{1} s_{2} \cdots s_{\ell}$. Then either no state is popped before the next execution of line 1 , and $\beta$ is of the form $\alpha s_{\ell+1}$, hence $\alpha \prec_{\operatorname{lex}} \beta$. Or $1 \leq k<\ell$ states are first popped, and the algorithm then pushes $t$ on the current path. By definition of the transition labeling $\lambda, t$ is a successor of $s_{\ell-k}$ such that $\lambda\left(s_{\ell-k}, s_{\ell-k+1}\right)<\lambda\left(s_{\ell-k}, t\right)$. Hence, the new value of $\mathrm{cp}$ is $\beta=s_{1} s_{2} \cdots s_{\ell-k} t$ and $\alpha \prec_{\text {lex }} \beta$.

Corollary 1. Algorithm 2 halts on any input.

Proof. There is a finite number of simple paths in a finite graph, $\mathrm{cp}$ takes its values in this finite set and each recursive call makes it greater.

Since Algorithm 2 discovers an increasing sequence of paths in the lexicographic ordering, it is natural to introduce the following sequence $\left(\gamma_{i}\right)_{0 \leq i \leq p}$. Let $\mathcal{S}$ be the finite set of simple accepting paths. Recall that a simple accepting path is of the form $\alpha s \beta s$ with $\alpha s \beta$ simple and $s \beta \cap F \neq \emptyset$. Since the lexicographic ordering is total, we can define a sequence $\left(\gamma_{i}\right)_{0 \leq i \leq p}$ as follows:

$$
\begin{cases}\gamma_{0}=\min _{\preceq_{\text {lex }}} \mathcal{S} & \text { if } \mathcal{S} \neq \emptyset \\ \gamma_{i+1}=\min _{\preceq_{\text {lex }}}\left\{\gamma \in \mathcal{S}|| \gamma|<| \gamma_{i} \mid\right\} & \text { if }\left\{\gamma \in \mathcal{S}|| \gamma|<| \gamma_{i} \mid\right\} \neq \emptyset\end{cases}
$$

where $|\gamma|$ denotes the length of $\gamma$. By construction, the last element $\gamma_{p}$ of this sequence is an accepting path of minimal length. Note that the sequence $\gamma_{0}, \ldots, \gamma_{p}$ is increasing in the lexicographic ordering and decreasing in length. For $\alpha, \beta \in \mathcal{S}$, we let $\alpha \sqsubseteq \beta$ if $\alpha \preceq$ lex $\beta$ and $|\alpha| \leq|\beta|$. We shall use the following simple fact. 
Fact 1 Each $\gamma_{i}$ is $\sqsubseteq$-minimal in $\mathcal{S}$.

Given a path $\alpha$, we let $\min (\alpha)=\min \left\{i \mid \alpha\right.$ is a prefix of $\left.\gamma_{i}\right\}$ and $\max (\alpha)=$ $\max \left\{i \mid \alpha\right.$ is a prefix of $\left.\gamma_{i}\right\}$. By convention, $\min (\alpha)=\infty$ and $\max (\alpha)=-\infty$ if $\alpha$ is not a prefix of some $\gamma_{i}$.

The next proposition implies in particular that Algorithm 2 is correct, since the last value taken by mce is precisely $\gamma_{p}$. It also shows what would be the behavior of a variant of our algorithm which outputs the successive values of mce. Although the time consumption of the algorithm is high, the algorithm can output all counterexamples of the sequence $\gamma_{i}$ when they are discovered, and the user can stop the search at any time. For instance, Dekker's algorithm produces about 80 counterexamples.

Proposition 2. The successive values taken by the variable mce during the execution of Algorithm 2 are $\gamma_{-1}=\varepsilon, \gamma_{0}, \ldots, \gamma_{p}$.

Proposition 2 is a direct consequence of Proposition 3 below. Indeed, DFS_MIN is initially called with the parameters $\left(s_{1}\right.$, normal) if $s_{1} \notin F$ and ( $s_{1}$, careful) if $s_{1} \in F$. If there exists a counterexample, the hypotheses of Proposition 3 are fulfilled at the beginning of the algorithm, with $s=s_{1}, \delta=\varepsilon$ and $k=0$. Hence, at the end of the initial call of DFS_MIN on $s_{1}$, the value of mce is $\gamma_{\max \left(s_{1}\right)}=\gamma_{p}$.

Proposition 3. Let $\delta s$ be a strict prefix of $\gamma_{k}$ with $k=\min (\delta s)<\infty$. Assume that at the beginning of a call DFS_MIN (s,mode), we have $\mathrm{cp}=\delta$ and that for all prefixes $\delta_{1} r$ of $\delta s$ we had mce $=\gamma_{\min \left(\delta_{1} r\right)-1}$ at the beginning of the call DFS_MIN $\left(r,{ }_{-}\right)$. Then, at the end of the call DFS_MIN (s, mode), we have $\mathrm{mce}=\gamma_{\max (\delta s)}$. Moreover, whenever the variable mce is updated, it is switched from some $\gamma_{\ell-1}$ to $\gamma_{\ell}$ with $\ell \geq 0$.

The proof of this proposition in turn uses Lemma 3.

Proof. Let $T=\{t \in E(s) \mid \min (\delta s t)<\infty\}$. Since $\delta s$ is a strict prefix of $\gamma_{\min (\delta s)}$, we have $T \neq \emptyset$. Write $T=\left\{t_{1}, \ldots, t_{n}\right\}$ with $\lambda\left(s, t_{i}\right)<\lambda\left(s, t_{i+1}\right)$ for all $1 \leq i<n$. We use an induction on $\left|\gamma_{k}\right|-|\delta s| \geq 1$.

Claim. If before line 4 when considering $t_{i} \in E(s)$ we have mce $=\gamma_{\min \left(\delta s t_{i}\right)-1}$ then after line 14 of this iteration we have mce $=\gamma_{\max \left(\delta s t_{i}\right)}$.

Let $t=t_{i}$ and $\ell=\min (\delta s t)$. Either $\ell=0$ and $\mathrm{mce}=\varepsilon$ or $|\mathrm{cp}|+1=|\delta s t| \leq$ $\left|\gamma_{\ell}\right|<|\mathrm{mce}|$ and the test line 4 succeeds.

The first case is when $t \in \mathrm{cp}=\delta s$. Then, we have $\gamma_{\ell}=\delta s t$ and $t$ closes an accepting path. Therefore, mce is updated to $\gamma_{\ell}$. For any other successor $v$ of $s$ with $\lambda(s, v)>\lambda(s, t)$, we have $|\delta s v|=\left|\gamma_{\ell}\right|=\mid$ mce $\mid$, hence the test line 4 fails. Therefore, the value of mce remains $\gamma_{\ell}$ until the end of the call DFS_MIN $\left(s,{ }_{-}\right)$. Moreover, from $\gamma_{\ell}=\delta s t$ we deduce that $i=n$ and $\gamma_{\ell}=\max \left(\delta s t_{n}\right)=\max (\delta s)$ which proves the claim.

The second case is when $t \notin \mathrm{cp}=\delta s$. All hypotheses of Lemma 3 are fulfilled, hence DFS_MIN $\left(t,,_{-}\right)$is called. When DFS_MIN $\left(t,,_{-}\right)$is called, $\delta s t$ is a strict prefix 
of $\gamma_{\ell}, \mathrm{cp}=\delta s$, mce $=\gamma_{\ell-1}=\gamma_{\min (\delta s t)-1}$ and for all prefixes $\delta_{1} r$ of $\delta s$ we had mce $=\gamma_{\min \left(\delta_{1} r\right)-1}$ at the beginning of the call DFS_MIN $\left(r,{ }_{-}\right)$. Therefore, the hypotheses of Proposition 3 are fulfilled and since $\left|\gamma_{\ell}\right|-|\delta s t|<\left|\gamma_{k}\right|-|\delta s|$ we get by induction that mce $=\gamma_{\max (\delta s t)}$ at the end of the call DFS_MIN $\left(t,{ }_{-}\right)$. The claim is proved.

Now, we show by induction on $i$ that before line 4 when considering $t_{i} \in E(s)$ we have mce $=\gamma_{\min }\left(\delta s t_{i}\right)-1$.

Note that $k=\min (\delta s)=\min \left(\delta s t_{1}\right)$. By definition of $\gamma_{k}$, no successor $t$ of $s$ with $\lambda(s, t)<\lambda\left(s, t_{1}\right)$ may be such that $\delta s t$ is on a simple accepting path of length less than $\left|\gamma_{k-1}\right|$ (with the convention $\left|\gamma_{-1}\right|=\infty$ ). Hence, the value of mce remains $\gamma_{k-1}$ until $t_{1} \in E(s)$ is considered. The property holds for $i=1$.

Assume now that the property holds for some $i<n$. From the claim, we get mce $=\max \left(\delta s t_{i}\right)$ after the iteration for $t_{i} \in E(s)$. Let $q=\max \left(\delta s t_{i}\right)$. Note that $\min \left(\delta s t_{i+1}\right)=\max \left(\delta s t_{i}\right)+1=q+1$. By definition of $\gamma_{q+1}$ and of the set $T$, no successor $v$ of $s$ with $\lambda\left(s, t_{i}\right)<\lambda(s, v)<\lambda\left(s, t_{i+1}\right)$ may be such that $\delta s v$ is on a simple accepting path of length less than $\left|\gamma_{q}\right|$. Hence, the value of mce remains $\gamma_{q}$ until $t_{i+1} \in E(s)$ is considered and the property still holds for $i+1$.

Finally, before line 4 when considering $t_{n} \in E(s)$ we have mce $=\gamma_{\min \left(\delta s t_{n}\right)-1}$. Using the claim, we get $\mathrm{mce}=\max \left(\delta s t_{n}\right)$ after the iteration for $t_{n} \in E(s)$. Note that $\max \left(\delta s t_{n}\right)=\max (\delta s)$. By definition of the set $T$, no successor $v$ of $s$ with $\lambda\left(s, t_{n}\right)<\lambda(s, v)$ may be such that $\delta s v$ is on a simple accepting path of length less than $\left|\gamma_{\max (\delta s)}\right|$. Hence, the value of mce remains $\gamma_{\max (\delta s)}$ until the end of DFS_MIN $(s$, mode $)$ and the proposition is proved.

The proof of the next lemma uses auxiliary results (Lemmas 5 and 6 below) on paths that are totally independent of the algorithm.

Lemma 3. Let $\delta$ st be a simple path with $\ell=\min (\delta s t)<\infty$. Assume that, while considering $t \in E(s)$ in DFS_MIN $\left(s,{ }_{-}\right)$, we have $\mathrm{cp}=\delta s$ and $\mathrm{mce}=\gamma_{\ell-1}$ and that for all prefixes $\delta_{1} r$ of $\delta s$ we had mce $=\gamma_{\min \left(\delta_{1} r\right)-1}$ at the beginning of the call DFS_MIN $\left(r,{ }_{-}\right)$. Then, DFS_MIN $\left(t,_{-}\right)$is called.

Proof. We let $\alpha^{\prime}=\delta s$. Assume first that $\ell=0$, so that $\alpha^{\prime} t$ is a prefix of $\gamma_{0}$. Since mce $=\gamma_{-1}=\varepsilon$, the test line 4 succeeds. Since $\alpha^{\prime} t$ is simple and $\mathrm{cp}=\alpha^{\prime}$, the test line 5 fails. Assume that the test line 7 fails. Then, the mode of the algorithm is necessarily normal, and in particular there is no accepting state on $\mathrm{cp}=\alpha^{\prime}$. Moreover, $t$ is not accepting: $\alpha^{\prime} t \cap F=\emptyset$. If the test line 9 also fails, then $t$ has already been visited along a simple path that we denote $\beta^{\prime} t$. By Lemma 2 , we have $\beta^{\prime} t \prec_{\text {lex }} \alpha^{\prime} t$. This situation is impossible by Lemma 6 . Hence the test line 9 must succeed and DFS_MIN $\left(t\right.$, , $\left._{-}\right)$is called in this case.

Assume now that $\ell \neq 0$. We have $|\mathrm{cp}|+1=\left|\alpha^{\prime} \mathrm{t}\right| \leq\left|\gamma_{\ell}\right|$ since $\alpha^{\prime} t$ is a prefix of $\gamma_{\ell}$. Further, $\left|\gamma_{\ell}\right|<\left|\gamma_{\ell-1}\right|$ by definition of the sequence $\left(\left|\gamma_{i}\right|\right)_{i}$. Since mce $=\gamma_{\ell-1}$, we deduce that the test line 4 succeeds. Since $\alpha^{\prime} t$ is simple and $\mathrm{cp}=\alpha^{\prime}$, the test line 5 fails. Assume that the test line 7 fails. We show as before that $\alpha^{\prime} t \cap F=\emptyset$. Assume that the test line 9 also fails. Then, there exists a simple path $\beta^{\prime} t$ such 
that $\beta^{\prime} t \prec_{\text {lex }} \alpha^{\prime} t$ and $\left|\beta^{\prime} t\right|=t \rightarrow$ depth. If $\left|\beta^{\prime} t\right|>\left|\gamma_{\ell}\right|$, then $t \rightarrow \operatorname{depth}=\left|\beta^{\prime} t\right|>$ $\left|\alpha^{\prime} t\right|=|\mathrm{cp}|+1$ and DFS_MIN $\left(t,{ }_{-}\right)$is called on line 11 .

Assume now that $\left|\beta^{\prime} t\right| \leq\left|\gamma_{\ell}\right|$. We want to apply Lemma 5 with $\alpha=\gamma_{\ell}$. Recall that $\gamma_{\ell}$ is a simple accepting path which is $\sqsubseteq$-minimal in $\mathcal{S}$. Note that $\alpha^{\prime} t$ is a prefix of $\alpha$ which satisfies property (1) of Lemma 5 and it remains to show that $\alpha^{\prime} t$ is minimal with this property. Since the algorithm is still in mode normal (the test line 7 failed), for all prefixes $\delta_{1} r$ of $\alpha^{\prime}$, we had $r \rightarrow$ depth $=\infty$ when the call to DFS_MIN $(r$, normal) was made. By hypothesis, at the beginning of this call, we had mce $=\gamma_{\ell_{1}-1}$ where $\ell_{1}=\min \left(\delta_{1} r\right)$. Assume that there is a simple path $\beta_{1}^{\prime} r \prec_{\text {lex }} \delta_{1} r$. Let $\beta_{1}^{\prime \prime} \sqsubseteq \beta_{1}^{\prime}$ be $\sqsubseteq$-minimal with this property. Since $\beta_{1}^{\prime \prime} r$ was not visited before $\delta_{1} r$, and since $\beta_{1}^{\prime \prime}$ is $\sqsubseteq$-minimal, we have $\left|\beta_{1}^{\prime} r\right| \geq\left|\beta_{1}^{\prime \prime} r\right| \geq\left|\gamma_{\ell_{1}-1}\right|>$ $\left|\gamma_{\ell}\right|=|\alpha|$ and property (1) of Lemma 5 does not hold for $\delta_{1} r$. Therefore, $\alpha^{\prime} t$ is the shortest prefix of $\alpha=\gamma_{\ell}$ satisfying (1) and Lemma 5 implies that $\left|\beta^{\prime}\right|>\left|\alpha^{\prime}\right|$. We conclude as above that $\operatorname{DFS} \_M I N\left(t,{ }_{-}\right)$is called on line 11 .

Lemma 4. Let $\delta=\alpha s \beta s$ be a path with $s \beta \cap F \neq \emptyset$, $\alpha$ s simple and $\alpha s \cap \beta=\emptyset$. We can construct a simple accepting path $\delta^{\prime}=\alpha^{\prime} s^{\prime} \beta^{\prime} s^{\prime}$ such that $\left|\delta^{\prime}\right| \leq|\delta|$ and $\alpha s$ is a prefix of $\alpha^{\prime} s^{\prime}$.

Proof. Assume that $\delta$ is not a simple accepting path. Let $t$ be the first state occuring twice in $\beta$. Then, we write $\beta=\beta_{1} t \beta_{2} t \beta_{3}$ with $t \notin \beta_{1} \beta_{2}$. If $s \beta_{1} t \beta_{3} \cap F \neq \emptyset$ then we let $\delta^{\prime}=\alpha s \beta_{1} t \beta_{3} s$. The path $\delta^{\prime}$ still satisfies the hypotheses of the lemma (with the same $\alpha$ and $s$ ) and we have $\left|\delta^{\prime}\right|<|\delta|$. Hence we can conclude by induction. Otherwise, we let $\delta^{\prime}=\alpha s \beta_{1} t \beta_{2} t$. Again, $\delta^{\prime}$ still satisfies the hypotheses of the lemma and $\left|\delta^{\prime}\right|<|\delta|$. Since $\alpha s$ is a prefix of $\alpha s \beta_{1} t$, we can again conclude by induction.

Lemma 5. Let $\alpha \in \mathcal{S}$ be a simple accepting path which is $\sqsubseteq$-minimal in $\mathcal{S}$. Assume that there exists a prefix $\alpha^{\prime} t$ of $\alpha$ satisfying

$$
\alpha^{\prime} t \cap F=\emptyset, \beta^{\prime} t \prec_{\text {lex }} \alpha^{\prime} t \text { and }\left|\beta^{\prime} t\right| \leq|\alpha| \text { for some simple path } \beta^{\prime} t .
$$

For the shortest prefix $\alpha^{\prime}$ t of $\alpha$ satsisfying (1) we have $\left|\beta^{\prime}\right|>\left|\alpha^{\prime}\right|$.

Proof. Let $\alpha_{1}$ be the greatest common prefix of $\alpha^{\prime}$ and $\beta^{\prime}$. We write $\alpha^{\prime}=\alpha_{1} \alpha_{2}$ and $\beta^{\prime}=\alpha_{1} \beta_{2}$. Note that the transition between the last state of $\alpha_{1}$ and the first state of $\beta_{2} t$ is strictly smaller than the transition between the last state of $\alpha_{1}$ and the first state of $\alpha_{2} t$. Hence, for all nonempty prefixes $\beta_{2}^{\prime}$ of $\beta_{2} t$ and $\alpha_{2}^{\prime}$ of $\alpha_{2} t$, we have $\alpha_{1} \beta_{2}^{\prime} \prec_{\text {lex }} \alpha_{1} \alpha_{2}^{\prime}$.

Assuming by contradiction that $\left|\beta_{2}\right| \leq\left|\alpha_{2}\right|$ we will build a simple accepting path $\beta$ with $\beta \prec_{\text {lex }} \alpha$ and $|\beta| \leq|\alpha|$, a contradiction with the $\sqsubseteq$-minimality of $\alpha$.

Write $\alpha=\alpha^{\prime} t \alpha^{\prime \prime}$ and let $s$ be the first state on $\beta_{2} t$ which occurs also on $t \alpha^{\prime \prime}$. We write $\beta_{2} t=\beta_{2}^{\prime} s \beta_{2}^{\prime \prime}$ and $\alpha^{\prime \prime}=\alpha_{3} s \alpha_{4}$ with $s \notin \alpha_{3}$. Below, whenever we state that a path is simple, this follows from the definition of $s$ and $\alpha_{3}$ and from the fact that $\alpha$ and $\beta^{\prime} t$ are simple.

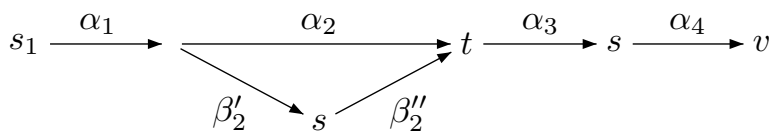


1. Assume that $\alpha_{3} s$ contains a final state. Then, $\delta=\alpha_{1} \beta_{2}^{\prime} s \beta_{2}^{\prime \prime} \alpha_{3} s$ is an accepting path which is not necessarily simple. Yet, $\beta^{\prime} t=\alpha_{1} \beta_{2}^{\prime} s \beta_{2}^{\prime \prime}$ is simple. Hence $\alpha_{1} \beta_{2}^{\prime} s$ is simple. Moreover, $\alpha_{1} \beta_{2}^{\prime} s \cap \beta_{2}^{\prime \prime} \alpha_{3}=\emptyset$ since $\alpha$ and $\beta_{2} t$ are simple and by definition of $s$ and $\alpha_{3}$. Applying Lemma 4, we obtain a simple accepting path $\beta$ with $|\beta| \leq|\delta| \leq|\alpha|$ and $\alpha_{1} \beta_{2}^{\prime} s$ is a prefix of $\beta$. We deduce that $\beta \prec_{\text {lex }} \alpha$ as announced.

Assume now that $\alpha_{3} s \cap F=\emptyset$, so that $\alpha_{4} \cap F \neq \emptyset$, and let $v$ be the last state of $\alpha$. By definition of an accepting path, $v$ is also the seed of the accepting loop. 2. We first show that $v$ does not occur in $\alpha_{2}$. Assume by contradiction that $\alpha_{2}=$ $\alpha_{2}^{\prime} v \alpha_{2}^{\prime \prime}$ and consider the simple path $\delta=\alpha_{1} \beta_{2}^{\prime} s \alpha_{4}=\delta^{\prime} v$. We have $\delta^{\prime} v \prec_{\text {lex }} \alpha_{1} \alpha_{2}^{\prime} v$ and $\left|\delta^{\prime} v\right| \leq|\alpha|$, a contradiction with the fact that $t$ is the first such state.

3. If $v$ does not occur in $t \alpha_{3}$ then we let $\beta=\alpha_{1} \beta_{2}^{\prime} s \alpha_{4}$.

4. If $v$ occurs in $t \alpha_{3}$ then we write $t \alpha_{3}=\alpha_{3}^{\prime} v \alpha_{3}^{\prime \prime}$ and we let $\beta=\alpha_{1} \beta_{2}^{\prime} s \alpha_{4} \alpha_{3}^{\prime \prime} s$.

In both cases, we can check that $\beta$ is a simple accepting path and that $\beta \prec_{\text {lex }} \alpha$ and $|\beta| \leq|\alpha|$ as desired.

Lemma 6. If $\alpha^{\prime} t$ is a prefix of $\gamma_{0}$ with $\alpha^{\prime} t \cap F=\emptyset$ then there is no simple path $\beta^{\prime} t$ with $\beta^{\prime} t \prec_{\text {lex }} \alpha^{\prime} t$.

Proof. Assume by contradiction that there exists a prefix $\alpha^{\prime} t$ of $\gamma_{0}$ such that $\alpha^{\prime} t \cap F=\emptyset$ and $\beta^{\prime} t \prec_{\text {lex }} \alpha^{\prime} t$ for some simple path $\beta^{\prime} t$. In the following, we assume that $\alpha^{\prime} t$ is the shortest such prefix of $\gamma_{0}$. We will build a simple accepting path $\beta$ with $\beta \prec_{\text {lex }} \gamma_{0}$, a contradiction with the definition of $\gamma_{0}$.

We proceed exactly as in the proof of Lemma 5 . The only difference is in case (2) when $v$ occurs in $\alpha_{2}$. Here we let $\beta=\alpha_{1} \beta_{2}^{\prime} s \alpha_{4} \alpha_{2}^{\prime \prime} t \alpha_{3} s$. Note that we may have $|\beta|>\left|\gamma_{0}\right|$ but we can show that $\beta$ is a simple accepting path with $\beta \prec_{\text {lex }} \gamma_{0}$, a contradiction with the $\preceq_{\text {lex }}$-minimality of $\gamma_{0}$.

\subsection{Remarks on the algorithm}

To keep the presentation simple, we have described the algorithm starting from a fresh input. However, one can also start from an automaton already tagged by Algorithm 1. Since no counterexample can go through a black state, this allows us to backtrack in the depth-first search as soon as a black state is seen. This shortens obviously the search by cutting useless parts of the automaton.

Moreover, one can also bound the search by the size of the counterexample produced by Algorithm 1. More precisely, Algorithm 2 is well suited for bounded model-checking. One can give it a bound $B$ for the depth of the research, and it would find successively the counterexamples $\gamma_{\ell}, \gamma_{\ell+1}, \ldots, \gamma_{p}$ where $\ell$ is the first index such that $\left|\gamma_{\ell}\right|<B$. This amounts only to changing the test of line 4 by

$$
(\text { mce }=\varepsilon \text { and }(\text { length }(\mathrm{cp})+1<B)) \text { or }(\text { length }(\mathrm{cp})+1<\text { length }(\text { mce }))
$$

\section{Implementation and experimental results}

The algorithm presented in Section 2 is quite efficient and visits each state at most twice (in view of Remark 1) in order to find a first counterexample. 
The second algorithm on the other hand finds the shortest counterexample at the expense of revisiting states much more often. In the worst case, its time complexity is exponential. In order to get the best of the two, we could start with the first algorithm until a first counterexample is found (if any) and then switch to the second algorithm to find the shortest counterexample.

In the prototype used to obtain the experimental results presented below, we actually used SPIN's algorithm for finding the first counterexample instead of our algorithm presented in Section 2. Then we switch to our minimization algorithm of Section 3. The reason is that more in-depth changes have to be carried out on SPIN's code to implement our algorithm of Section 2 and our primary goal was just to minimize the size of the counterexample. We are currently implementing the algorithm of Section 2 and since it is always more efficient than SPIN's one, more improvements can be expected.

In the synchronized product between the model and the LTL automaton built by SPIN, there is a strict alternation between transitions of the model and transitions of the LTL automaton (see [9]). Therefore all accepting paths are of odd length and when minimizing the size of a counterexample we can replace line 4 of Algorithm 2 by length (cp) $+2<$ length(mce). The test line 4 works in fact for an arbitrary Büchi automaton. This trivial optimization is important for our algorithm since it may revisit states quite often.

We have conducted experiments for various algorithms and specifications. Experiments for which the model does not satisfy the specification and counterexamples exist are gathered in Table 1. We compare our algorithm with SPIN -i

\begin{tabular}{|c|l|c|c|}
\cline { 3 - 4 } \multicolumn{2}{c|}{} & SPIN -i & Contrex \\
\hline \multirow{3}{*}{ Peterson } & counterexample length & 55 & 19 \\
& states stored & 80 & 85 \\
& states matched & 1968 & 9469 \\
& computation time & $0.030 \mathrm{~s}$ & $0.070 \mathrm{~s}$ \\
\hline \multirow{3}{*}{ Dijkstra } & counterexample length & 173 & 23 \\
& states stored & 539 & 543 \\
& states matched & 48593 & $2.5 * 10^{6}$ \\
& computation time & $0.240 \mathrm{~s}$ & $11.420 \mathrm{~s}$ \\
\hline \multirow{3}{*}{ Hyman $)$} & counterexample length & 5 & 5 \\
& states stored & 211258 & 209687 \\
& states matched & $1.96928 \mathrm{e}+09$ & 654246 \\
& computation time & $71 \mathrm{~m} 27.700 \mathrm{~s}$ & $1.780 \mathrm{~s}$ \\
\hline & counterexample length & 97 & 17 \\
& states stored & 123 & 157 \\
& states matched & 7389 & 40913 \\
& computation time & $0.080 \mathrm{~s}$ & $0.210 \mathrm{~s}$ \\
\hline
\end{tabular}

Table 1. Experiments for various algorithms when a counterexample does exist

which tries to reduce the size of the counterexample. Clearly SPIN -i does not 
find the shortest counterexample while we have proved in Section 3 that our algorithm does. The automata of the specifications (never-claims) have been generated by the tool LTL2BA [6] both for the verification with SPIN -i and with our algorithm. For each experiment, we show, in addition to the size of the minimal counterexample found, the number of different states visited by the algorithms (states stored). The last information (states matched) is the number of states (re)visited during the algorithm. Here, each time a state is (re)visited this counter is incremented. The execution time which is indicated is the user time (the system time is negligible in all cases) obtained on a Pentium III 700Mhz with $1 \mathrm{~Gb}$ of $\mathrm{RAM}$ and $1 \mathrm{~Gb}$ of cache.

As expected, our algorithm needs in general to revisit more states that SPIN's in order to really find the minimal counterexample. Yet, there are cases where SPIN's algorithm is less efficient than ours. The reason is that SPIN -i does not test whether a counterexample already exists (test $\mathrm{mce} \neq \varepsilon$, line 11 of Algorithm 2). For Dijkstra's algorithm, there is no counterexample in the left part of the graph. Therefore, until the first counterexample has been found, our algorithm does not switch to careful mode, even if a state's depth gets lowered.

\section{Conclusion and open problems}

The main contribution of this paper is the algorithm presented in Section 3 which finds a shortest accepting path in a Büchi automaton. It actually finds a sequence of counterexamples of decreasing length which it can output. It has been implemented and the comparison with SPIN's algorithm clearly demonstrates its superiority in counterexample length. We also proposed an algorithm to find a counterexample, without trying to minimize its length, which is more efficient than SPIN's. It avoids unnecessary revisits of states and hence finds a counterexample more quickly. We plan to implement this algorithm and to compare it experimentally with SPIN. Further, this algorithm detects states that cannot be part of an accepting path (black states). Hence, using it instead of SPIN's before searching for a minimal counterexample should improve the performance of our second algorithm.

Finding a shortest counterexample with a depth-first search algorithm is time consuming because we need to revisit states many times. A general goal for improving the efficiency is to detect more states that need not be revisited.

Another important issue is to be able to deal with partial order reductions. While the first nested-DFS algorithm [5] failed in the presence of partial order reductions, the version of [10] is able to cope with some reductions. We need to investigate whether our algorithms can handle partial order reductions with reasonable memory requirements.

Finally, it would be interesting to find ways to minimize the length of the counterexample with respect to the model and the LTL specification. Instead, existing algorithms search for counterexamples for the model and a specific automaton associated with the LTL specification. It is often the case that this 
specific automaton is not optimal for finding a short counterexample for the LTL formula.

Acknowledgment. The authors wish to thank the anonymous referees for their careful reading of the submitted version of the paper and for their comments.

\section{References}

1. E. M. Clarke, O. Grumberg, S. Jha, Y. Lu, and H. Veith. Counterexample-guided abstraction refinement. In Proceedings of the 12th Conference on Computer Aided Verification ( $C A V^{\prime} 01$ ), volume 1855 of $L N C S$, pages 154-169. Springer, 2001.

2. E. M. Clarke, O. Grumberg, and D. A. Peled. Model checking. MIT Press, 1999.

3. E.M. Clarke, O. Grumberg, S. Jha, Y. Lu, and H. Veith. Counterexample-guided abstraction refinement for symbolic model checking. J. ACM, 50(5):752-794, 2003.

4. Th. H. Cormen, C. Stein, R. L. Rivest, and Ch. E. Leiserson. Introduction to Algorithms. McGraw-Hill Higher Education, 2001.

5. C. Courcoubetis, M. Vardi, P. Wolper, and M. Yannakakis. Memory efficient algorithms for the verification of temporal properties. In Computer-aided verification '90 (New Brunswick, NJ, 1990), volume 3 of DIMACS Ser. Discrete Math. Theoret. Comput. Sci., pages 207-218. Amer. Math. Soc., Providence, RI, 1991.

6. P. Gastin and D. Oddoux. Fast LTL to Büchi automata translation. In G. Berry, H. Comon, and A. Finkel, editors, Proceedings of the 13th Conference on Computer Aided Verification ( $\left.C A V^{\prime} 01\right)$, number 2102 in Lect. Notes Comp. Sci., pages 53-65. Springer, 2001.

7. T.A. Henzinger, R. Jhala, R. Majumdar, and G. Sutre. Lazy abstraction. In Proc. of POPL'02, pages 58-70. ACM Press, 2002.

8. G. Holzmann. The model checker spin. IEEE Trans. on Software Engineering, 23(5):279-295, May 1997. Special issue on Formal Methods in Software Practice.

9. G. Holzmann. The SPIN model-checker. Addison-Wesley, 2003.

10. G. Holzmann, D. Peled, and M. Yannakakis. On nested depth first search. In Proc. Second SPIN Workshop, pages 23-32, Rutgers, Piscataway, NJ, 1996. American Mathematical Society.

11. M. Y. Vardi and P. Wolper. An automata-theoretic approach to automatic program verification. In Proc. 1st Symp. on Logic in Computer Science, pages 332-344, Cambridge, June 1986. 